\title{
Propiedades psicométricas de la Escala de Bienestar Psicológico SPWB de Carol d. Riff en estudiantes de secundaria de la UGEL 02
}

\author{
Psychometric properties of the SPWB Psychological Well-being Scale of \\ Carol d. Riff in secondary school students of the UGEL 02
}
Patricia del Pilar Díaz Gamarra ${ }^{1}$, Erika Roxana Estrada Alomía ${ }^{2}$, Noemí Edith Iparraguirre Yaurivilca ${ }^{3}$ Universidad César Vallejo

Alex Teófilo Grajeda Montalvo ${ }^{4}$

Universidad Nacional Mayor de San Marcos

Miguel Ángel Misare Condori ${ }^{5}$ ESSALUD

Recibido: $13-09-20$

Aceptado: $16-11-20$

Publicado: $21-12-20$

\section{Resumen}

Se estudiaron las propiedades psicométricas de la Escala de Bienestar Psicológico SPWB de Riff en escolares secundarias de la UGEL 02. Con dicho fin y trabajando con 1190 adolescentes, que fueron seleccionados bajo muestreo probabilístico, se administró dicho instrumento.. Se redujo la escala de 34 a 12 ítems tomando como criterio la correlación ítem-test $\geq .30$, y la tabla factorial $\geq .40$. Se verificó la pertinencia del modelo de cuatro factores para medir bienestar psicológico en adolescentes: Autonomía, Crecimiento, Propósito y Autoaceptación. La consistencia interna cuantificada con los coeficientes Omega y Alpha fueron de 0.86 y 0.72 , considerándose aceptable. Se elaboraron normas de interpretación basadas en rangos percentilares.

Palabras clave: Bienestar psicológico; consistencia interna; análisis factorial confirmatorio.

1 Docente en Universidad César Vallejo.

Autor para correspondencia: gpatriciad@ucv.edu.pe ORCID: https://orcid.org/0000-0002-2003-2849

2 Docente en Universidad César Vallejo.

E-mail: eestradaal@ucvvirtual.edu.pe ORCID: https://orcid.org/0000-0001-5916-1034

3 Docente en Universidad César Vallejo.

E-mail: niparraguirrey@ucv.edu.pe ORCID: https://orcid.org/0000-0003-3167-6807

4 Docente asociado, Universidad Nacional Mayor de San Marcos. Lima, Perú.

E-mail: agrajedam@unmsm.edu.pe ORCID: https://orcid.org/0000-0001-5972-2639

5 Psicólogo en ESSALUD.

E-mail: miguelmisare@gmail.com ORCID: https://orcid.org/0000-0002-4749-1260

(C) Los autores. Este artículo es publicado por la Revista de Investigación en Psicología de la Facultad de Psicología, Universidad Nacional Mayor de San Marcos. Este es un artículo de acceso abierto, distribuido bajo los términos de la licencia Creative Commons Atribucion - No Comercia_Compartir Igual 4.0 Internacional. (http://creativecommons.org/licenses/by-nc-sa/4.0/) que permite el uso no comercial, distribución y reproducción en cualquier medio, siempre que la obra original sea debidamente citada. 


\begin{abstract}
This research presents the Psychometric Properties of the Riff SPWB Psychological Wellbeing Scale in secondary students of UGEL 02 . It was administered to a sample consisting of 1188 adolescents, chosen by a probabilistic sampling. The objective of the present investigation was to describe the psychometric properties of the Riff SPWB Psychological Well-being Scale in secondary students of UGEL 02. The scale was reduced from 34 to 12 items based on the item-test correlation $\geq .30$, and the factor table $\geq .40$. The relevance of the four-factor model to measure psychological well-being in adolescents was verified: Autonomy, Growth, Purpose and Self-acceptance. The internal consistency quantified with the Omega coefficient was 0.86 and for Alpha was 0.72 , considered acceptable. Interpretation standards based on percentile ranks were developed.
\end{abstract}

Keywords: Psychological well-being; internal consistency; Confirmatory factor analysis.

El mundo actual exige a las personas el manejo apropiado de un conjunto de capacidades, habilidades y herramientas personales que les permitan adaptarse a las necesidades de la sociedad actual; mostrando autonomía, capacidad de constituir relaciones interpersonales positivas y el establecimiento de metas que permita su crecimiento en todo ámbito en el proceso de su autorrealización.

En ese sentido Ryff en 1989a propone una teoría multidimensional, considerando el crecimiento personal y las capacidades para un desarrollo positivo; reforzando el potencial del individuo que está relacionado con la autorrealización.

Durante la adolescencia varios aspectos juegan un papel importante: el familiar, con los roles y jerarquías que el adolescente observa e introyecta; el grupo de pertenencia, las relaciones con sus pares y la toma de consciencia de su propia autoestima. Es por ello que velar por el bienestar psicológico de los adolescentes, es una labor integral y necesaria dentro de la educación, que debe asegurar la formación integral, por tanto, los adolescentes puedan sentirse valorados, autónomos, interactúen adecuadamente con su entorno y establezcan metas para su crecimiento personal. En relación a esto, Ryff (1989a) menciona la importancia de cuidar el bienestar psicológico, de los adolescentes, mediante el desarrollo de recursos propios que permitan hacerle frente a situaciones que impliquen un desequilibrio emocional. Las personas estables emocionalmente, se caracterizan por ser muy empáticos y con adecuados sentimientos hacia las demás personas, asimismo tienen grandes facultades para mantener relaciones de amor y amistad sólidas, colocando a la variable bienestar psicológico como un indicadito deseable de lo saludable que se encontraría mentalmente (Oramas, Santana y Vergara, 2003).

En las instituciones educativas del cono norte de Lima metropolitana se observan estudiantes con dificultades conductuales, emocionales y familiares; tales como, déficits en las habilidades sociales, baja autoestima, desesperanza aprendida, 
ansiedad, disfuncionalidad familiar y ausencia total o parcial de metas a futuro, que impiden el alcance de la autorrealización y el bienestar psicológico.

Para la presente investigación se tomará lo expuesto por Riff (2013) y Riff \& Singer (2013) debido a que se presenta al bienestar psicológico de manera integral y subdividido en 6 dimensiones: Auto-aceptación, autonomía, relaciones positivas, propósito de vida, dominio del entorno, y crecimiento personal, que permite un análisis global y parcial de la variable. Este modelo es de gran utilidad, porque nos proporciona un indicador de bienestar psicológico individual y a la vez aplicado en grupo, permite determinar los diversos niveles de desarrollo al interior del mismo. Por otro lado, estos resultados brindarán una perspectiva más amplia para trabajar resiliencia, crecimiento personal y fortalecimiento de habilidades intrapersonales e interpersonales en adolescentes para que alcancen el bienestar psicológico necesario para un desarrollo adecuado y exitoso.

Para explorar esta variable existen diversos instrumentos psicológicos; sin embargo, no se cuenta con alguno que abarque nuestro contexto en la población considerada en la presente investigación; debido a ello y para realizar lo planteado se analizará la Escala de Bienestar Psicológico SPWB por lo que es válido preguntarse

¿Cuáles son las propiedades psicométricas de la Escala de Bienestar Psicológico SPWB de Riff en estudiantes de secundaria de la UGEL 02, 2019?

Para lograr ello nos planteamos como objetivos 1. Describir las características psicométricas del SPWB de Riff en estudiantes que cursan la secundaria en la UGEL 02, 2019, 2. Determinar los índices de confiabilidad, 3. Determinar y describir las evidencias de validez de constructo de las dimensiones de la escala y 4. Elaborar los baremos de la Escala de Bienestar Psicológico SPWB de Riff para los estudiantes de instituciones educativas estatales de la UGEL 02.

El ejecutar esta investigación permitirá contar con un instrumento que nos permita identificar en que medida se encuentra el bienestar psicológico de estudiantes secundarios que asisten a escuelas de tres distritos que pertenecen al cono norte asegurando que se pueda tener un diagnóstico cercano a nuestra realidad y plantear las medidas preventivas y correctivas de ser necesario; asegurando la estabilidad psicológica, la autonomía, auto aceptación y propósito de vida en los escolares, formando así ciudadanos que otorguen un impacto positivo en su vida y por ende en el desarrollo de la sociedad.

\section{Antecedentes}

\section{Internacionales}

Pineda, Castro, Chaparro (2017) desarrollaron un estudio psicométrico de la escala de bienestar psicológico de Ryff en adultos jóvenes colombianos. La finalidad 
de la investigación fue evaluar la confiabilidad y la validez de constructo de la escala en mención. Tuvo como muestra a 727 jóvenes seleccionados por un muestreo no probabilístico. Como resultados obtuvieron una validez de constructo que se ejecutó mediante el AFC con índices de ajuste adecuados $(\mathrm{X} 2=1649.40, \mathrm{gl}=362$, $\mathrm{p}=0.00 ; \mathrm{CFI}=0.95 ; \mathrm{AGFI}=0.95 ; \mathrm{RMSEA}=0.066, \mathrm{IC} 90 \%, 0.062-0.069 ; \mathrm{SRMR}$ $=0.077$ ) en los seis factores y un factor de segundo orden denominado Bienestar; la confiabilidad que se realizó mediante el coeficiente de Omega obtuvo valores entre 0.60 y 0.84 . El resultado que considera esta investigación fue que la escala es apropiada para la evaluación de bienestar psicológico en la muestra planteada.

Freire, Ferradás, Núnez y Valle (2017) en España estudial la estructura factorial de la escala Bienestar psicológico de Ryff; su grupo de aplicación fueron 1402 estudiantes universitarios realizando un análisis factorial confirmatorio a través el paquete estadístico SPSS 21 y AMOS 21. Los hallazgos muestran que un modelo de 4 factores tienen mejores indicadores de ajuste que los datos teóricos, siendo los factores: autoaceptación, dominio del entorno, propósito en la vida y crecimiento personal. Asimismo, la confiabilidad general del instrumento fue mesurada mediante el coeficiente de alfa obteniendo .87 considerada más que aceptable.

\section{Nacionales}

Samanez, Alva y Jaimes (2016) efectuaron una investigación sobre el bienestar psicológico y la asertividad en estudiantes de secundaria de una institución educativa privada de Lima Este, la cual tuvó como objetivo evaluar la relación existente entre las variables bienestar psicológico y asertividad. Su muestra de estudio fue de 221 estudiantes y como resultado general obtuvieron que estas dos variables guardan una r parcial, siendo mayor la fuerza en los casos donde es mayor el establecimiento de satisfactorias relaciones sociales.

Vallejos (2015) desarrolló una investigación que relaciona la satisfacción con la vida y el bienestar psicológico, en estudiantes de $4^{\circ}$ y $5^{\circ}$ año de secundaria del distrito de Los Olivos, los instrumentos que utilizó fueron: la Escala de Calidad de Vida de Olson \& Barnes para adolescentes y la escala BIEPS-J para adolescentes y jóvenes. Los resultados señalan: Que los estudiantes adolescentes de 4to y 5to año de secundaria de colegios estatales del distrito de Los Olivos presentan un nivel de bienestar psicológico promedio y de calidad de vida. Asimismo, existe relación significativa entre calidad de vida y bienestar psicológico, lo cual nos indica que a mayor calidad de vida, mayor es el bienestar psicológico.

\section{Teoría Positiva del Bienestar Psicológico}

Definición de Bienestar Psicológico: Dentro de los modelos de la psicología positiva Carol Riff entre los años 1989 - 1998 plantea un modelo de bienestar psicológico que parte de elementos placenteros que son parte del funcionamiento óptimo del ser humano ya que le producen más emociones positivas. Por ende, el 
bienestar psicológico del individuo se rige por los diferentes retos a los que se enfrenta de acuerdo a las fases del ciclo de la vida en el que está involucrando y de forma paralela a su felicidad y placer (Ryff y Singer, 1998).

Por otro lado, Chávez (2006), determina el bienestar psicológico como la apreciación subjetiva que exterioriza la satisfacción de las personas y su nivel de agrado con aspectos particulares o generales de su vida en los que predomina el estado de ánimo positivo y relacionado con componentes afectivos y cognitivos.

Dentro de todo esto es importante definir el bienestar psicológico a partir de elementos no hedónicos, es decir que no incluye la cantidad de emociones positivas o de placer como predictor en uno mismo del funcionamiento psicológico óptimo sino más bien como una consecuencia.

Esta propuesta se relaciona con la concepción de bienestar como virtud, ya que va en la búsqueda constante de la excelencia personal, el modelo propone el desarrollo del bienestar psicológico en el transcurso de la vida mediante metas que permitan alcanzar el verdadero potencial de cada uno, la autorrealización (Riff, 1989b).

\section{Dimensiones del bienestar psicológico}

De acuerdo al modelo planteado para la variable "bienestar psicológico" cada dimensión que forma parte de este constructo indica un índice de bienestar, encontrando las siguientes dimensiones:

\section{Autoaceptación}

Se basa en la capacidad de la persona de conocerse a sí mismo, trabajando de una manera idónea para descubrir sus emociones, motivaciones y acciones; englobando de manera general la perspectiva que uno tiene sobre sus gustos y las cosas que no sean de su agrado, diferenciarlas de los demás para reconocer y aprobar sus fortalezas y debilidades como seres únicos e individuales. Asimismo, es sirve como indicativo sobre la salud mental, la posibilidad de realizarse, el óptimo funcionamiento y el grado madurativo social. Esto implica que la persona este satisfecha consigo misma y con sus las experiencias de vida es decir, la autoaceptación de los atributos personales reflejando más que una adecuada autoestima (Keyes, Ryff y Shmotkin, 2002).

\section{$\underline{\text { Relaciones positivas }}$}

Las personas como seres sociales que son necesitan establecer relaciones con los demás que propicien vínculos que generen confianza, y desarrollen aquella que capacidad que permita amar. Estas relaciones positivas son las relaciones interpersonales de confianza y expresiones de amor a los demás, esta capacidad establece 
una relación inversa en la que disminuye el estrés e incrementa el bienestar psicológico (Ryff, 1989a).

\section{Autonomía}

La autonomía proporciona a las personas la capacidad de regular adecuadamente sus comportamientos y poder sobrellevar la influencia social. Esta se relaciona entre la voluntad y la acción, es decir, la persona se siente libre de realizar las conductas que elija. Para lograr plena autonomía se requiere una autonomía física y funcional que permita tomar decisiones libremente elegidas (Ryff y Keyes, 1995).

\section{$\underline{\text { Dominio del Entorno }}$}

Permite desarrollar la capacidad de reconocer y manejar situaciones dificultosas mediante un adecuado funcionamiento físico y mental. De esa manera, generar y escoger áreas satisfactorias para los deseos o necesidades que presente cada persona, al tener este control de su ambiente contribuyen positivamente en el mismo. Esta es la habilidad que tiene la persona para poder elegir y crear entornos adecuados para satisfacer sus necesidades y para desarrollarse óptimamente, es la habilidad del individuo para hacer usos de sus talentos e intereses y la participación activa en el entorno que brinden funciones psicológicas positivas (Keyes et al, 2002).

\section{Propósito de vida}

Está orientada hacia una perspectiva positiva donde se busca dar un propósito y sentido a la vida proponiéndose metas, y determinando sus objetivos; ya que esos elementos son fundamentales para una adecuada salud mental en el ser humano. Es así que para un óptimo funcionamiento es preciso que la persona se proponga sus propias metas, objetivos a obtener en la vida, además de contar con el sentido de dirección e intencionalidad en la vida (Keyes, et al, 2002).

\section{$\underline{\text { Crecimiento personal }}$}

Promueve el desarrollo potencial de la persona y del desarrollo de sus capacidades, consolidando competencias individuales. Un óptimo bienestar psicológico requiere también que la persona se esfuerce por ir creciendo y desarrollando al máximo todas sus habilidades, capacidades con logros obtenidos en el pasado y afronte nuevos retos personales que le permitan crecer (Ryff y Singer, 2008).

\section{Teoría clásica de los tests}

En la presente investigación se ha usado la teoría clásica en virtud a que "históricamente es la más antigua y además se considera que su uso es predominante tanto en la construcción como en el análisis de las propiedades psicométricas de los tests psicológicos" (Grajeda, 2018, p.18). 


\section{Supuestos de la Teoría clásica}

Para Cortada De Cohan (1999) y Muñiz (2010) la teoría clásica está basada en el modelo lineal clásico que fuera presentado y sustentado por Spearman.

Primer supuesto: El modelo considera que la puntuación empírica es el puntaje obtenido por el usuario que ha rendido la prueba. Sin embargo esta PE tendría dos sub elementos.

\section{$\underline{\text { Sub elementos de la puntuación empírica: }}$}

1. La puntuación verdadera del examinado (signada con V)

2. El error (signado con e). Dicho sub elemento es producto de diferentes factores en el examen.

Segundo supuesto: La puntuación verdadera y el error no están correlacionados.

Tercer supuesto: Los errores que presentan las personas en dos tests no están correlacionados.

\section{Adaptación de un test o escala}

De acuerdo con Vijver y Leung, Citado por Mikulic, para adaptar un test o escala psicológica existen tres niveles; el primero de ellos implica la traducción del mismo de un idioma hacia otro y su aplicación, en el segundo nivel se ven las modificaciones de los ítems considerando la cultura y contexto en que se utilizarán, y finalmente en el tercer nivel se realiza un nuevo instrumento ya que la mayor parte de los ítems necesitan una rigurosa modificación (2017, p.42).

\section{Análisis Factorial Exploratorio}

De acuerdo con Pérez, Chacón y Moreno el análisis factorial exploratorio tiene como finalidad delimitar la cantidad de factores que debe tener un constructo mediante análisis estadísticos que establezcan parámetros para ello (2000, párr. 7- 8).

\section{Análisis Factorial Confirmatorio}

Para la realización del análisis factorial confirmatorio se debe contar con una base teórica pertinente y profunda sobre la variable ya que esta evaluará si los datos planteados realmente se ajustan a la teoría realizando una seria de hipótesis utilizando matrices de varianza y covarianza para analizar y confirmar su estructura (Fernández, 2015, párr. 11 -13).

\section{Estadísticos de confiabilidad}

\section{Coeficiente Alfa}

Estima la confiabilidad interna de un instrumento; dentro del área de ciencias sociales su uso es muy recurrente y se considera tanto en la construcción como en 
la verificación de propiedades psicométricas de un instrumento (Cervantes, 2005, párr. 23-25).

\section{Coeficiente Omega}

Se realiza mediante el análisis factorial de los ítems y para poder estimarlo se requiere previamente el análisis factorial confirmatorio ya que este utiliza las cargas factoriales, Gerbing. \& Anderson (1988).

\section{Baremos}

Según Abad, Garrido, Olea y Ponsola, los baremos de un test son puntuaciones directas obtenidas mediante la aplicación en un instrumento, otorgando a cada posible resultado directo un valor numérico que indica el lugar que ocupa en relación a los valores obtenidos por las personas que componen el grupo normativo (2006, pp.119 - 124).

\section{MÉTODO}

Se ha utilizado un enfoque cuantitativo para nuestra investigación que es considerada de tipo instrumental ya que en este tipo se diseñan y validan pruebas para estimar la presencia de una variable, Montero y León (2002).

La población estudiantil de secundaria de al UGEL 02 está compuesta por 50918 estudiantes matriculados en secundaria pertenecientes a colegios estatales de los distritos del Rímac, Los Olivos y San Martín de Porres. Las edades fluctuaron entre los 11 y 19 años. El $55 \%$ estuvo compuesto por varones y el $45 \%$ por mujeres.

Para determinar el tamaño muestral con un 95 por ciento de confianza y un riesgo de error de 3 se ha usado la fórmula para poblaciones finitas mencionada por Abad y Servin (1981, en Grajeda, 2018, p. 37), obteniéndose como resultado 1046, sin embargo, para evitar la muerte experimental se aplicó el instrumento a 1250 estudiantes, quedando 1190 protocolos llenados correctamente los que finalmente conformaron la muestra. Previamente se utilizó un muestro probabilístico aleatorizado y por conglomerados para seleccionar la muestra, Sánchez y Reyes (2015).

Instrumento: Escala de Bienestar Psicológico SPWB de Ryff

\section{Ficha técnica:}

Nombre original

Año

Autores

Objetivo
: Escala de Bienestar Psicológico SPWB de Ryff

: 1995

: Carol Ryff

: Evaluar bienestar psicológico 


$\begin{array}{ll}\text { Administración } & \text { : Individual o Colectiva } \\ \text { Adaptación peruana } & : \text { Zavala y Silva (adaptación) } \\ \text { Estructura } & : 6 \text { dimensiones (34 ítems) } \\ \text { Escala tipo } & \text { : Likert } \\ \text { Tiempo de administración } & \text { : Aproximadamente } 30 \text { minutos. } \\ \text { Significación } & : \text { Evalúa bienestar psicológico } \\ \text { Presenta las siguientes dimensiones: } & \\ \text { Dimensión 1: Autoaceptación } & \\ \text { Dimensión 2: Relaciones positivas } & \\ \text { Dimensión 3: Autonomía } & \\ \text { Dimensión 4: Dominio del Entorno } \\ \text { Dimensión 5: Propósito de vida } \\ \text { Dimensión 6: Crecimiento personal }\end{array}$

Validez y Confiabilidad: Pineda, Castro, Chaparro (2017) trabajando con estudiantes colombianos y realizando análisis factorial encuentra los 6 factores propuestos y uno de segundo orden al que denominan Bienestar. En relación a la confiabilidad estos autores encuentran valores Omega entre 0.60 y 0.84 .

De otro lado en Perú Briones (2019) trabajando con la versión abreviada aplicada a adolescentes cajamarquinos encuentra una estructura de 5 factores al realizar el análisis factorial. En relación a la confiabilidad obtiene un alfa de 0.83 . También se aplicó test retest con un coeficiente igual a 0.63 .

\section{Procedimiento}

Los datos se recogieron aplicando el instrumento en las aulas de los estudiantes a los que se informó de los fines del estudio, garantizándoles la confidencialidad al tratar sus datos.

\section{Análisis de datos}

La información recogida se procesó estadísticamente con la teoría clásica de los tests obteniéndose estadísticos descriptivos. Luego se procedió a estudiar la validez de constructo con el análisis factorial exploratorio (AFE), posteriormente se determinó la confiabilidad mediante las pruebas alfa de Cronbach y Omega. Paso seguido se estudió la normalidad de las puntuaciones con la prueba KS no encontrándose normalidad por lo que procedió a estudiar las diferencias significativas según sexo con las prueba U de Mant Witney y por edad con la prueba de K Wa1lis, calculándose un NS igual a 0.117 en el primer caso y de 0,087 en el segundo, no encontrándose diferencias significativas ni por sexo ni por edad, por lo que se elaboró un solo baremo. Los programas usados han sido SPSS 26 y Jamovi. 


\section{RESULTADOS}

Se consideraron 12 ítems, tres por cada una de las cuatro dimensiones, tomando en cuenta la correlación ítem-test corregida $\geq .30$, y la tabla factorial $\geq .40$ (Kline, 2015). Así mismo, se decidió eliminar las dimensiones Relaciones positivas y Dominio del entorno dado que la mayoría de sus ítems no cumple con los criterios.

Tabla 1

Análisis estadístico preliminar de items

\begin{tabular}{|c|c|c|c|c|c|c|c|c|c|c|c|c|c|}
\hline \multirow{2}{*}{ Dimensión } & \multirow{2}{*}{ Ítems } & \multicolumn{4}{|c|}{ FR } & \multirow{2}{*}{$\mathbf{M}$} & \multirow{2}{*}{ DE } & \multirow{2}{*}{$\mathbf{g}^{1}$} & \multirow{2}{*}{$\mathrm{g}^{2}$} & \multirow{2}{*}{ IHC } & \multirow{2}{*}{$\mathbf{h}^{2}$} & \multirow{2}{*}{ id } & \multirow{2}{*}{ Aceptable } \\
\hline & & 1 & 2 & 3 & 4 & & & & & & & & \\
\hline \multirow{6}{*}{ Autoaceptación } & B1 & 5.0 & 17.8 & 45.3 & 31.8 & 3.0 & 0.8 & -0.6 & -0.2 & 0.43 & 0.40 & 0.00 & Sí \\
\hline & B7 & 3.1 & 7.0 & 18.6 & 71.3 & 3.6 & 0.8 & -1.9 & 2.7 & 0.58 & 0.60 & 0.00 & Sí \\
\hline & B12 & 10.3 & 18.5 & 43.3 & 27.9 & 2.9 & 0.9 & -0.5 & -0.5 & 0.42 & 0.34 & 0.00 & No \\
\hline & B16 & 3.1 & 7.6 & 21.9 & 67.3 & 3.5 & 0.8 & -1.7 & 2.1 & 0.58 & 0.61 & 0.00 & Sí \\
\hline & B22 & 11.0 & 24.6 & 34.9 & 29.5 & 2.8 & 1.0 & -0.4 & -0.9 & 0.27 & 0.15 & 0.00 & No \\
\hline & B28 & 4.3 & 11.0 & 23.4 & 61.3 & 3.4 & 0.8 & -1.3 & 0.8 & 0.54 & 0.56 & 0.00 & Sí \\
\hline \multirow{5}{*}{$\begin{array}{l}\text { Relaciones } \\
\text { positivas }\end{array}$} & B2 & 7.2 & 12.9 & 30.3 & 49.6 & 3.2 & 0.9 & -1.0 & 0.0 & 0.26 & 0.62 & 0.00 & No \\
\hline & B8 & 10.9 & 17.7 & 32.4 & 38.9 & 3.0 & 1.0 & -0.6 & -0.7 & 0.25 & 0.60 & 0.00 & No \\
\hline & $\mathrm{B} 13$ & 3.7 & 9.3 & 35.8 & 51.2 & 3.3 & 0.8 & -1.1 & 0.8 & 0.29 & 0.49 & 0.00 & No \\
\hline & B23 & 3.5 & 11.0 & 28.7 & 56.7 & 3.4 & 0.8 & -1.2 & 0.7 & 0.41 & 0.61 & 0.00 & Sí \\
\hline & B29 & 4.4 & 8.7 & 26.3 & 60.6 & 3.4 & 0.8 & -1.4 & 1.2 & 0.31 & 0.49 & 0.00 & Sí \\
\hline \multirow{5}{*}{ Autonomía } & B3 & 10.1 & 23.0 & 38.8 & 28.1 & 2.8 & 0.9 & -0.4 & -0.7 & 0.24 & 0.68 & 0.00 & Sí \\
\hline & B4 & 13.9 & 21.8 & 33.0 & 31.3 & 2.8 & 1.0 & -0.4 & -1.0 & 0.32 & 0.61 & 0.00 & Sí \\
\hline & B9 & 12.2 & 22.0 & 33.7 & 32.1 & 2.9 & 1.0 & -0.4 & -0.9 & 0.25 & 0.54 & 0.00 & Sí \\
\hline & B18 & 4.5 & 14.5 & 36.1 & 44.9 & 3.2 & 0.9 & -0.9 & 0.0 & 0.27 & 0.66 & 0.00 & No \\
\hline & B24 & 13.4 & 30.3 & 33.9 & 22.3 & 2.6 & 1.0 & -0.1 & -1.0 & 0.27 & 0.36 & 0.00 & No \\
\hline \multirow{6}{*}{$\begin{array}{l}\text { Dominio del } \\
\text { entorno }\end{array}$} & B5 & 10.8 & 30.9 & 37.2 & 21.0 & 2.7 & 0.9 & -0.2 & -0.8 & 0.14 & 0.48 & 0.00 & No \\
\hline & B10 & 5.6 & 13.9 & 40.4 & 40.1 & 3.1 & 0.9 & -0.8 & 0.0 & 0.25 & 0.44 & 0.00 & No \\
\hline & B14 & 12.1 & 22.6 & 39.9 & 25.4 & 2.8 & 1.0 & -0.4 & -0.8 & 0.15 & 0.45 & 0.00 & No \\
\hline & B19 & 8.7 & 28.1 & 40.1 & 23.1 & 2.8 & 0.9 & -0.3 & -0.7 & 0.17 & 0.61 & 0.00 & No \\
\hline & B25 & 3.7 & 14.8 & 43.9 & 37.6 & 3.2 & 0.8 & -0.7 & 0.0 & 0.35 & 0.53 & 0.00 & $\mathrm{Si}$ \\
\hline & B32 & 10.4 & 19.0 & 36.3 & 34.3 & 2.9 & 1.0 & -0.6 & -0.7 & 0.12 & 0.33 & 0.00 & No \\
\hline \multirow{5}{*}{$\begin{array}{l}\text { Propósito de } \\
\text { vida }\end{array}$} & B6 & 7.3 & 16.2 & 41.0 & 35.5 & 3.0 & 0.9 & -0.7 & -0.3 & 0.33 & 0.43 & 0.00 & Sí \\
\hline & B11 & 1.3 & 6.5 & 33.6 & 58.7 & 3.5 & 0.7 & -1.2 & 1.2 & 0.36 & 0.49 & 0.00 & Sí \\
\hline & B15 & 8.1 & 15.1 & 35.6 & 41.2 & 3.1 & 0.9 & -0.8 & -0.3 & 0.32 & 0.38 & 0.00 & No \\
\hline & B20 & 2.9 & 5.7 & 20.8 & 70.6 & 3.6 & 0.7 & -1.9 & 3.1 & 0.37 & 0.48 & 0.00 & Sí \\
\hline & B26 & 15.3 & 30.8 & 31.8 & 22.0 & 2.6 & 1.0 & -0.1 & -1.0 & 0.11 & 0.05 & 0.00 & No \\
\hline \multirow{5}{*}{$\begin{array}{l}\text { Crecimiento } \\
\text { personal }\end{array}$} & B17 & 1.9 & 7.0 & 36.9 & 54.3 & 3.4 & 0.7 & -1.2 & 1.1 & 0.34 & 0.63 & 0.00 & si \\
\hline & B21 & 2.3 & 7.2 & 29.9 & 60.7 & 3.5 & 0.7 & -1.4 & 1.6 & 0.33 & 0.65 & 0.00 & si \\
\hline & B27 & 21.3 & 29.5 & 28.5 & 20.7 & 2.5 & 1.0 & 0.0 & -1.2 & 0.02 & 0.76 & 0.00 & No \\
\hline & B30 & 12.0 & 19.4 & 26.7 & 42.0 & 3.0 & 1.0 & -0.6 & -0.9 & 0.22 & 0.45 & 0.00 & No \\
\hline & B31 & 4.5 & 9.4 & 38.6 & 47.5 & 3.3 & 0.8 & -1.1 & 0.7 & 0.26 & 0.37 & 0.00 & si \\
\hline
\end{tabular}

Nota: FR: Formato de respuesta; M: Media; DE: Desviación estándar; $\mathrm{g}^{1}$ : coeficiente de asimetría de Fisher; $\mathrm{g}^{2}$ : coeficiente de curtosis de Fisher; IHC: Índice de homogeneidad corregida; $\mathrm{h}^{2}$ : Comunalidad; ID: Índice de discriminación. 


\section{Análisis factorial confirmatorio}

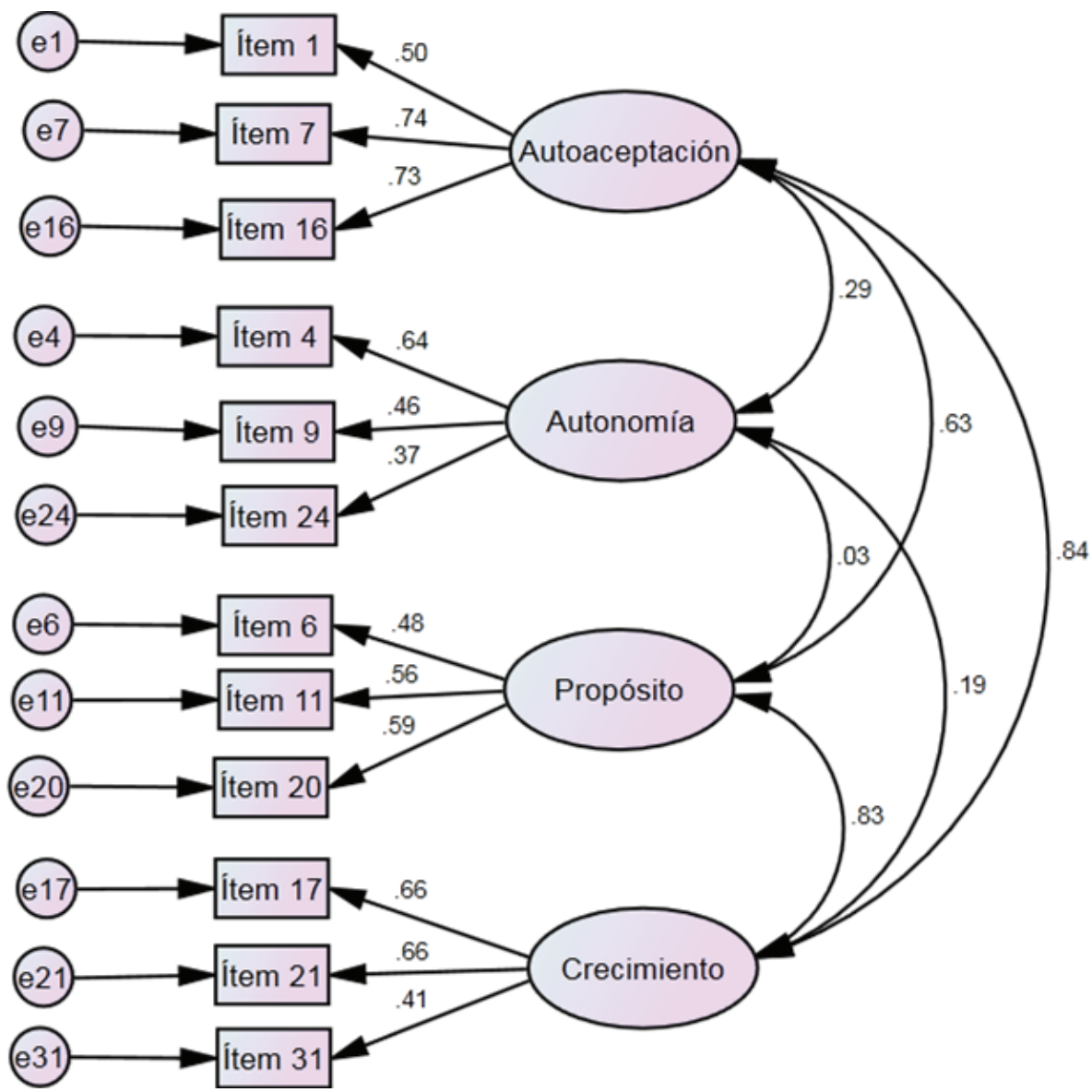

Figura 1. Modelo de cuatro factores correlacionados

Luego de realizar el análisis factorial confirmatorio se encontraron índices de ajustes aceptables: Índices de ajuste: $\mathrm{CFI}=.96, \mathrm{TLI}=.95, \mathrm{SRMR}=.02, \mathrm{RM}-$ $\mathrm{SEA}=.03$ (Brown, 2015)

Tabla 2

Coeficientes de consistencia interna

\begin{tabular}{lccc}
\hline & Alfa de Cronbach & Omega de McDonald & N de elementos \\
\hline Bienestar psicológico & 0.72 & 0.86 & 12 \\
Autoaceptación & 0.68 & 0.70 & 3 \\
Autonomía & 0.48 & 0.50 & 3 \\
Propósito & 0.55 & 0.56 & 3 \\
Crecimiento & 0.60 & 0.63 & 3 \\
\hline
\end{tabular}


La magnitud del coeficiente de Alfa de Cronbach y de Omega de Mc Donald evidencia niveles aceptables de fiabilidad para la prueba, sin embargo, las puntuaciones las dimensiones deben ser interpretadas con precaución.

En la tabla 3 podemos notar que al ser 0.117 el NS entonces se infiere que no hay diferencias significativas entre varones y mujeres.

Tabla 3

Estudio de las diferencias por sexo

\begin{tabular}{|c|c|c|c|c|}
\hline \multicolumn{5}{|c|}{ Rangos } \\
\hline & Sexo & $\mathbf{N}$ & Rango promedio & Suma de rangos \\
\hline \multirow{3}{*}{ Bienestar } & Masculino & 656 & 609,07 & 399547,50 \\
\hline & Femenino & 533 & 577,69 & 307907,50 \\
\hline & Total & 1189 & & \\
\hline \multicolumn{2}{|c|}{ U de Mann-Whitney } & \multicolumn{3}{|c|}{165596,500} \\
\hline \multicolumn{2}{|c|}{ W de Wilcoxon } & \multicolumn{3}{|c|}{307907,500} \\
\hline \multicolumn{2}{|c|}{$\mathrm{Z}$} & \multicolumn{3}{|c|}{$-1,568$} \\
\hline \multicolumn{2}{|c|}{ Sig. asintót. (bilateral) } & \multicolumn{3}{|c|}{, 117} \\
\hline
\end{tabular}

En la tabla 4 podemos notar que al ser 0.87 el NS entonces se infiere que no hay diferencias significativas entre las distintas edades.

Tabla 4

Estudio de las diferencias edad a partir de la prueba de Kruskal Wallis

\begin{tabular}{lccc}
\hline & \multicolumn{3}{c}{ Rangos } \\
\hline & Edad & N & Rango promedio \\
\hline & 11 & 20 & 655,75 \\
& 12 & 172 & 597,73 \\
& 13 & 252 & 642,61 \\
Bienestar & 14 & 282 & 590,13 \\
& 15 & 236 & 598,83 \\
& 16 & 170 & 541,36 \\
& 17 & 45 & 528,99 \\
& 18 & 11 & 538,00 \\
Chi-cuadrado & 19 & 1 & 98,00 \\
Gl & Total & 1189 & \multicolumn{3}{c}{13,799} \\
Sig. asintót. & & \multicolumn{3}{c}{, 087} \\
\hline
\end{tabular}

Tabla 5

Baremos total y por dimensiones

\begin{tabular}{ccccccc}
\hline Percentiles & $\begin{array}{c}\text { Bienestar } \\
\text { psicológico }\end{array}$ & Autoaceptación & Autonomía & $\begin{array}{c}\text { Propósito de } \\
\text { vida }\end{array}$ & $\begin{array}{c}\text { Crecimiento } \\
\text { personal }\end{array}$ & Niveles \\
\hline 25 & $12--35$ & $3--8$ & $3-7$ & $3--8$ & $3--8$ & Bajo \\
50 & $36--41$ & $9--10$ & $8-9$ & $9--10$ & $9--10$ & Promedio \\
75 & $42--48$ & $11--12$ & $10--12$ & $11--12$ & $11--12$ & Alto \\
\hline
\end{tabular}




\section{DISCUSION}

Para esta investigación se tomó el Modelo Teórico de Bienestar psicológico de Riff, adaptado por Leal y Silva (2010), el cual considera seis factores: Autoaceptación, Autonomía, Propósito de vida, Crecimiento personal, Relaciones positivas y Dominio del entorno; luego de realizar el AFC y verificar las correlaciones ítemtest, se encontró evidencia del ajuste del modelo de cuatro factores: Autonomía, Crecimiento, Propósito y Autoaceptación, debido a que obtuvieron coeficientes $\geq$ .30 , y la tabla factorial $\geq .40$ (Kline, 2015). Asimismo, se decidió eliminar las dimensiones Relaciones positivas y Dominio del entorno dado que la mayoría de sus ítems no cumple con los criterios. De similar forma el trabajo de Freire, Ferradás, Núnez y Valle (2017) encontraron cuatro factores pero solo se coincide en tres de ellos los cuales son, Crecimiento, Propósito y Autoaceptación.

La estimación del CFI, que realiza la comparación entre el modelo propuesto y el modelo nulo, determina la ajuste dependencia entre los factores, cuanto más cercano es éste valor a 1 indica la idoneidad del modelo propuesto frente al nulo; el CFI obtenido en este estudio es de .96, aceptándose el modelo propuesto (Hair, Anderson, Tatham \& Black, 1999). Pineda, Castro, Chaparro (2017) también encuentra un CFI bastante cercano el cual fue de 0.95 .

En cuanto a los valores del Error de Aproximación Cuadrático medio (RMSEA), en general, cuando son inferiores a .5 señalan un buen ajuste, en nuestro estudio el valor encontrado fue de 0.3 (Browne y Cudeck, 1993). En ese sentido el trabajo de Pineda, Castro, Chaparro (2017) realizado con jóvenes colombianos presenta un mejor RMSEA ya que fue de 0.066

El coeficiente de Omega $(\omega)$, que estima la consistencia interna basada en las cargas factoriales, diversos autores indican que se debe utilizar este coeficiente frente a otros por ser un estimador más sensible (Zinbarg, Revelle, Yovel y Li, 2005). En el presente estudio el Coeficiente omega $(\omega=86)$ considerado como bueno, ligeramente superior al encontrado en el Coeficiente de Alfa de Cronbach que fue de .72. Coeficientes similares, entre 0.83 y 0.87, han sido hallados por Pineda, Castro, Chaparro (2017), Freire, Ferradás, Núnez y Valle (2017); y Briones (2019).

Se recomienda el uso de las puntuaciones percentilares integradas, dado que no se hallaron diferencias por sexo y edad y asimismo sefacilita la interpretación de los resultados obtenidos en la aplicación de la escala, a la vez que el uso del percentil es adecuado para variables psicológicas como lo es el bienestar psicológico.

\section{CONCLUSIONES}

La estructura de la Escala de bienestar psicológico quedaría configurada por los factores Autonomía, Crecimiento, Propósito y Autoaceptación. 
La Escala de bienestar psicológico de Carol D. Riff es altamente confiable.

La Escala de bienestar psicológico de Carol D. Riff posee muy buena validez de constructo.

Los estudiantes varones y mujeres no presentan diferencias significativas en la variable bienestar psicológico.

Los estudiantes no presentan diferencias significativas en la variable bienestar psicológico en función a la edad.

Se propone un baremo integrado sin diferencias de sexo ni edad.

\section{Agradecimientos / Acknowledgments:}

Los autores agradecen el apoyo brindado por los Directores de las IE participantes de la UGEL 02: IE N² 2031 "Virgen de Fátima", IE "República de Colombia", IE N 3014 "Leoncio Prado", IE "Palmas Reales", IE "Independencia”, quienes autorizaron la realización del presente estudio en sus instalaciones.

\section{Fuentes de financiamiento / Funding:}

Financiado por Fondos concursables para proyectos de investigación, desarrollo e innovación 2019 de la Universidad César Vallejo, aprobado por Resolución de Vicerrectorado de Investigación Nº12-2019-VI-UCV, del 22 de marzo del 2019.

\section{Rol de los autores / Authors Roles:}

PDG: realizó el diseño de investigación, responsable de los análisis temáticos, resultados de la investigación y redacción del artículo.

EEA: realizó el analista temático y co-redactor del artículo.

NIY: realizó la recolección de la muestra y procesamiento de datos.

AGM: realizó la corrección de formato y estilo, procesador y analista de datos.

MMC: realizó la recolección de la muestra

\section{Aspectos éticos / legales; Ethics / legals:}

Los autores declaran no haber incurrido en aspectos antiéticos respetando los códigos de investigación con humanos reseñados en el código de Ética profesional del Colegio de psicólogos del Perú.

Se solicitó autorización al Director de la IE N² 2031 "Virgen de Fátima", IE "República de Colombia", IE No 3014 "Leoncio Prado", IE "Palmas Reales", IE "Independencia" Los participantes firmaron el consentimiento informado el cual brindaba datos de la naturaleza, medios y objetivos de la investigación . 
Se ha respetado lo establecido por las normativas éticas que regulan el ejercicio profesional (Código de Ética del Colegio de Psicólogos del Perú) Se procuró la protección de la confidencialidad de la información personal e institucional, asegurando el anonimato de las personas e instituciones involucradas en la muestra.

\section{Conflicto de intereses / Competing interests:}

Los autores manifestamos no haber tenido ningún conflicto de intereses al realizar esta investigación y articulo.

\section{REFERENCIAS}

Abad, F., Garrido, J., Olea, J., y Ponsoda, V. (2006). Introducción a la Psicometría. Teoría Clásica de los Test y Teoría de la Respuesta al Ítem. Madrid: Universidad Autónoma.

Briones, K. (2019). Propiedades psicométricas de la escala de bienestar psicológico (SPWB) de Ryff versión abreviada en adolescentes de la ciudad de Cajamarca. (Tesis para optar por el título profesional de Licenciada en Psicología). Universidad Privada Antonio Guillermo Urrelo, Cajamarca. Recuperado de http://repositorio.upagu. edu.pe/handle/UPAGU/813

Browne MW, Cudeck R. (1993). Alternative Ways of Assessing Model Fit. In: Bollen K, Long J, editors. Testing Structural Equation Models. Sage; Newbury Park, CA:. pp. $136-162$.

Brown, T.A. (2015). Confirmatory factor analysis for applied research. New York: Guilford Publications.

Chavez, S. (2008). Bienestar psicológico en practicantes de yoga. (Tesis para optar por el título de Licenciado en Psicología con mención en Psicología Clínica). Pontificia Universidad Católica del Perú, Lima. Recuperado de http://tesis.pucp.edu.pe/repositorio/handle/123456789/627

Cervantes, V. (2005). Interpretaciones del coeficiente alpha de Cronbach. Avances en medición, 3, 9-28. Recuperado de http://www.humanas.unal.edu.co/psicometria/files/1113/8574/8604/Articulo_1_Alfa_de_Cronbach_9-28_2.pdf

Cortada de Cohan, N. (1999). Teoría psicométricas y construcción de tests. Buenos Aires: Lugar Editorial.

Fernández, A. (2015). Aplicación del análisis factorial confirmatorio a un modelo de medición del rendimiento académico en lectura. Ciencias económicas, 33 (2), 39.66. DOI: http://dx.doi.org/10.15517/rce.v33i2.22216

Freire, C., Ferradás, M., Núnez, J. y Valle, A. (2017). Estructura factorial de las Escalas de Bienestar Psicológico de Ryff en estudiantes universitarios. European Journal of Education and Psychology. 10, 1-8. https://doi.org/10.1016/j.ejeps.2016.10.001

Gerbing, D. W. \& Anderson J. C. (1988). An update paradigm for scale development incorporating unidimentionality and its assessment. Journal of Marketing Research, 25 (2), pp. 186-192. https://doi.org/10.1177/002224378802500207 
Grajeda, A. (2018). Construcción y validación de un test de aptitud funcional selectora visual en estudiantes de escuelas estatales del primer grado de primaria del distrito de Jesús María, 2018. (Tesis doctoral). Recuperado de http://repositorio.ucv.edu.pe/ bitstream/handle/UCV/ 21249/Grajeda_MAT.pdf? sequence=1\&isAllowed=y

Hair, J., Anderson, R., Tatham, R., \& Black, W. (1999). Análisis Multivariante. Madrid: Prentice Hall

Keyes, C., Ryff, C., y Shmotkin, D. (2002). Optimizing well-being: theempirical encounter of two traditions. Journal of Personality and Social Psychology, 82, 1007-1022

Kline, R. B. (2015). Principles and practice of structural equation modeling. New York: Guilford Publications.

Leal, R. y Silva, R. (2010). El bienestar psicológico del adolescente cajamarquino: adaptación local de la escala SPWB de Ryff. Cajamarca: Universidad Privada Antonio Guillermo Urrelo.

Montero, I., \& León, O. G. (2002). Clasificación y descripción de las metodologías de investigación en psicología. International Journal of Clinical and Health Psychology, 2(3), 503- 508.

Mikulic, I. M. (2017). Construcción y Adaptación de pruebas psicológicas. Archivos de trabajos de la Universidad de Buenos Aires Facultad de Psicología. Obtenido de http://23118.psi.uba.ar/academica/carrerasdegrado/psicologia/informacion_adicional/obligatorias/059_psicometricas1/tecnicas_psicometricas/archivos/f2.pdf

Muñiz, J. (2010). Las teorías de los tests: teoría clásica y teoría de respuesta a los ítems. Papeles del Psicólogo 31 (1) pp. 57-66. Recuperado el 01/04/2016 en http://webcache. googleusercontent.com/search?q=cache:-KnC2SAmEl8J:www.papelesdelpsicologo. es/pdf/1796.pdf $+\& \mathrm{~cd}=3 \& \mathrm{hl}=$ es $\& \mathrm{ct}=\mathrm{clnk} \& \mathrm{gl}=$ pe

Oramas, A.; Santana, S. y Vergara, A. (2006). El bienestar psicológico, un indicador de salud mental positivo. Revista Cubana de Salud y Trabajo, 7(1-2), 34-39

Pérez, J., Chacón, S., y Moreno, R. (noviembre, 2000). Validez de constructo: el uso de análisis factorial exploratorio-confirmatorio para obtener evidencias de validez. España. Revista Psicothema. Recuperado de http://www.redalyc.org/html/727/72797102/

Pineda, C., Castro, J., y Chaparro, R. (2017). Estudio psicométrico de las Escalas de Bienestar Psicológico de Ryff en adultos jóvenes colombianos. Pensamiento Psicológico, 16(1), 44-55. doi:10.11144/Javerianacali.PPSI16-1.epeb

Ryff, C. (1989a). Happiness is everything, or is it? Explorations on the meaning of psychological well-being. Journal of Personality and Social Psychology, 57(6), 1069-1081. https://doi.org/10.1037/0022-3514.57.6.1069.

Ryff, C. (1989b). Beyond Ponce de Leon and life satisfaction: New directions in quest of successful aging. International Journal of Behavioral Development, 12(1), 35-55. https://doi.org/10.1177/016502548901200102.

Ryff, C., y Keyes, L. (1995). The structure of psychological well-being revisited. New York: Journal of Personality and Social Psychology, 69(4), 719-727. http://dx.doi. org/10.1037/0022-3514.69.4.71 
Ryff, C., y Singer, B. (1998). The contours of positive health. New York: Psychological Inquiry.

Ryff, C. D., \& Singer, B. H. (2008). Know thyself and become what you are: A eudaimonic approach to psychological well-being. Journal of Happiness Studies: An Interdisciplinary Forum on Subjective Well-Being, 9(1), 13-39. https://doi.org/10.1007/s10902006-9019-0.

Ryff, C. D. (2013). Eudaimonic well-being and health: Mapping consequences of selfrealization. In A. S. Waterman (Ed.), The best within us: Positive psychology perspectives on eudaimonia (p. 77-98). American Psychological Association. https://doi. org/10.1037/14092-005

Ryff, C. D., \& Singer, B. H. (2013). Know thyself and become what you are: A eudaimonic approach to psychological well-being. In A. Delle Fave (Ed.), Happiness studies. The exploration of happiness: Present and future perspectives (p. 97-116). Springer Science + Business Media. https://doi.org/10.1007/978-94-007-5702-8_6

Samanez, A., Alva, L. y Jaimes, J. (2016). Bienestar psicológico y asertividad en estudiantes de secundaria de una institución educativa privada de Lima Este, 2016. Recuperado de: https://www.researchgate.net/publication/320392181_Bienestar_ psicologico_y_asertividad_en_estudiantes_de_secundaria_de_una_institucion_ educativa_privada_de_Lima_Este_2016

Sánchez, H. y Reyes, C. (2015). Metodología y diseños en la investigación científica. Lima: Universidad Ricardo Palma.

Vallejos. M. (2015). Calidad de Vida y Bienestar Psicológico en adolescentes estudiantes de 4to y 5 to año de educación secundaria del distrito de Los Olivos. Tesis para optar el grado académico de Licenciado en Psicología. Lima, Perú: Universidad César Vallejo.

Zinbarg, R.E., Revelle, W., Yovel, I. \& Li W. (2005). Cronbach's $\alpha$, Revelle's $\beta$, and Mcdonald's $\omega \mathrm{H}$ : their relations with each other and two alternative conceptualizations of reliability. Psychometrika 70, 123-133. https://doi.org/10.1007/s11336-0030974-7 
